\title{
PARALLEL VECTOR FIELDS AND THE TOPOLOGY OF MANIFOLDS
}

\author{
BY LEON KARP 1
}

Communicated by E. H. Brown, Jr., July 20, 1976

0. On a closed, differentiable $n$-manifold $M$ there exists a nowhere vanishing vector field if and only if the Euler-Poincare characteristic is zero, $\chi(M)=0$. If $M$ is riemannian, one may ask whether or not it admits a vector field that is parallel. Chern has shown that necessary conditions are that the first Betti number $b_{1} \geqslant 1$ and that the second betti number $b_{2} \geqslant b_{1}-1$, and he has conjectured that these conditions are not sufficient [4]. It follows from a special case of a subsequent result of Bott [2] that all the Pontryagin numbers of such a manifold are zero. This is a restriction only if dimension $M \equiv 0 \bmod 4$. The purpose of this note is to announce some further necessary topological conditions. We also exhibit a family of manifolds with $\chi=0$ that satisfy the conditions of Chern and Bott but still cannot admit parallel vector fields whatever the metric. If the manifold is complex we can refine our results to deduce additional conditions that are necessary for the existence of a Kähler-parallel vector field. These results are applied to the topology of compact homogeneous spaces (supplementing some similar results of Hurewicz and de Rham). Finally, we give some $n$ dimensional generalizations of some classical results of Hurwitz on Riemann surfaces of genus $\geqslant 2$.

1. Let $M^{n}$ be an $n$-dimensional, closed, differentiable manifold and let $b_{j}(M) \equiv j$ th Betti number of $M$.

THEOREM A. If $M^{n}$ admits a vector field that is parallel with respect to some riemannian metric then the Betti numbers of $M$ satisfy:

$$
b_{1} \geqslant 1 \text { and } b_{k+1} \geqslant b_{k}-b_{k-1} \text {, for } 1 \leqslant k \leqslant n-1 .
$$

Notice that when $k=1$ we have Chern's condition: $b_{2} \geqslant b_{1}-1$.

Let $M$ be a closed, complex $n$-manifold. Then the Hodge number $h^{p, q}(M)$ is the dimension of $H^{q}\left(M, \Omega^{p}\right)$. For a Kähler manifold it is known that $\Sigma_{p+q=j} h^{p, q}(M)=b_{j}(M)$ and we can refine Theorem A.

AMS (MOS) subject classifications (1970). Primary 53C20, 53C30; Secondary 57F15.

1 These results form a portion of the author's doctoral dissertation written under the supervision of Professor L. Nirenberg, to whom the author would like to express his gratitude and appreciation. 
THEOREM B. If a closed complex n-manifold $M$ admits a vector field that is parallel with respect to a Kähler metric then the Hodge numbers of $M$ satisfy

$$
h^{p+1, q} \geqslant h^{p, q}-h^{p-1, q} \text { for } 0 \leqslant p \leqslant n, 0 \leqslant q \leqslant n .
$$

The manifolds $S(p, q, r) \equiv S^{1} \times S^{p} \# S^{q} \times S^{r}$ with $3 \leqslant q<r-1$, and $p+1=q+r \equiv 1 \bmod 2$, satisfy the conditions of Chern and Bott but still do not admit parallel vector fields for any metric. It is easy to construct other examples.

2. It is known that the Betti numbers of compact homogeneous spaces satisfy: (a) $b_{j} \leqslant(\underset{j}{\operatorname{dim}})$ (de Rham) and (b) if $b_{1} \neq 0$ then $b_{j} \geqslant\left(\begin{array}{c}b \\ j\end{array}\right)$ (Hurewicz). (See [6].)

COROLlaRY C. If $G / H$ is a compact homogeneous space with $b_{1} \neq 0$ then the Betti numbers of $G / H$ satisfy $b_{k+1} \geqslant b_{k}-b_{k-1}$ for all $1 \leqslant k \leqslant n-1$.

3. A classical theorem of Hurwitz [7] may be restated as: "A closed twodimensional manifold of genus $\geqslant 2$ has a finite isometry group for any riemannian structure, and it admits no one parameter group of holomorphic transformations for any complex structure" cf. [1]. If a two-dimensional manifold has Gauss curvature $K(x) \leqslant 0$ (and $\neq \equiv$ ) then $1 / 2 \chi=$ arithmetic genus $=\left(h^{0,0}-h^{0,1}\right)=1$ - genus $<0$ i.e. $b_{2}<b_{1}-1$ or $0=h^{0,2}<h^{0,1}-h^{0,0}$. Bochner [1] has extended Hurwitz' theorem to $n$-manifolds under the hypothesis of Ricci curvature $<0$ in place of $K(x) \leqslant 0$ or genus $\geqslant 2$. By explicitly introducing topological conditions (in place of using the Gauss-Bonnet theorem) we can get the same result for Ricci $\leqslant 0$.

THEOREM D. If a closed riemannian manifold $\left(M^{n}, g\right)$ has Ricci $\leqslant 0$ and satisfies $b_{k+1}<b_{k}-b_{k-1}$ for some $1 \leqslant k \leqslant n-1$, then the isometry group of $(M, g)$ is finite.

THEOREM D'. If a closed Kühler manifold has Ricci $\leqslant 0$ and satisfies $h^{p, q+1}<h^{p, q}-h^{p, q-1}$ for some $0 \leqslant p \leqslant n, 1 \leqslant q \leqslant n$ then it admits no connected group of holomorphic transformations.

For a manifold with real dimension $=2$ these conditions on the Betti and Hodge numbers mean genus $\geqslant 2$. If we strengthen our hypothesis we can prove:

THEOREM E. If a closed riemannian manifold $(M, g)$ has nonpositive sectional curvature and Betti numbers satisfying $b_{k+1}<b_{k}-b_{k-1}$ for some $1 \leqslant$ $k \leqslant n-1$ then $\operatorname{Diff}(M)^{0}$ (the identity component of the group of diffeomorphisms of $M$ ) contains no nontrivial isometries.

For $n=2$, Theorem $\mathrm{E}$ is also due to Hurwitz [2]. See also [5].

4. Finally, we have topological obstructions to nonnegative curvature:

THEOREM F. If a closed n-manifold $M$ has nonzero first Betti number and 
$b_{k+1}<b_{k}-b_{k-1}$ for some $1 \leqslant k \leqslant n-1$ then it admits no riemannian structure with Ricci $\geqslant 0$.

Theorems A and F may be applied to finite coverings of $M$. Also, assuming the validity of a well-known conjecture of Calabi [3], Theorem F implies topological conditions for Kähler manifolds with vanishing canonical class.

Proofs, examples, and related results will appear in [9].

\section{BIBLIOGRAPHY}

1. S. Bochner, Vector fields and Ricci curvature, Bull. Amer. Math. Soc. 52 (1946), 776-797. MR 8, 230.

2. R. Bott, Vector fields and characteristic numbers, Michigan Math. J. 14 (1967), 231-244. MR 35 \#2297.

3. E. Calabi, On Kähler manifolds with vanishing canonical class, Algebraic Geometry and Topology, Princeton Univ. Press, Princeton, N. J., 1957, pp. 78-89. MR 19, 62.

4. S. S. Chern, The geometry of G-structures, Bull. Amer. Math. Soc. 72 (1966), 167-219. MR 33 \#661.

5. T. Frankel, On theorems of Hurwitz and Bochner, J. Math. Mech. 15 (1966), 373-377. MR 33 \#675.

6. H. Hopf and H. Samelson, Ein Satz über die Wirkungsräume geschlossener Liescher Gruppen, Comment. Math. Helv. 13 (1941), 240-251. MR 4, 3.

7. A. Hurwitz, Über algebraische Gebilde mit eindeutigen Transformationen in sich, Math. Ann. 41 (1893), 403-442.

8. - Über diejenigen algebraischen Gebilde welche eindeutige Transformationen in sich zulassen, Math. Ann. 32 (1887), 290-308.

9. L. Karp, Vector fields on manifolds, Thesis, New York Univ., 1976.

DEPARTMENT OF MATHEMATICS, COURANT INSTITUTE OF MATHEMATICAL SCIENCES, NEW YORK UNIVERSITY, NEW YORK 10012 